WS10-C02

\title{
The Application of the Microseismic Monitoring in Natural Fracture Detection
}

\author{
C. Yin* (Sichuan Geophysical Company of CNPC), F.R. Wu (Sichuan \\ Geophysical Company of CNPC), Y.L. Li (Sichuan Geophysical Company of \\ CNPC), H. Liu (Sichuan Geophysical Company of CNPC), G.M. He (Sichuan \\ Geophysical Company of CNPC) \& C.H. Chen (Sichuan Geophysical \\ Company of CNPC)
}

\section{SUMMARY}

Microseismic monitoring (MSM) is one of the effective and real-time evaluation techniques for the fracturing. The results from in-field real-time MSM always provides the valuable benefits that help us gain an insight into the changes in the reservoir and the status of the fracturing operation. Sichuan area has a complex structure characteristic and many natural fractures are activated by the fracturing. The paper describes the different fracturing responses between the shale and tight sand, reveals its special characteristic of the natural fracture which activated by fracturing and probably provides the method (Relative magnitude), suitable analysis (Radiated pattern, Linear distribution), and interpretation (Integrated fracture interpretation) to detect the natural fracture through MSM and prevent induced fracture propagation towards fault zones. 


\section{Amsterdam ' 14}

\section{Introduction}

Microseismic monitoring (MSM) is one of the effective and real-time evaluation techniques for the fracturing. The distribution of the MSM events will play an important role in the interpretation of the hydro-fracture from the MSM signal received. Through the real time distribution of the MSM origin magnitude in 3D space, it will contribute to the analysis of the fracture tendency which stimulated by the fracturing, and directs fracture operation, and eventually better directs the exploitation of the unconventional oil and gas. The results from in-field real-time MSM always provides the following detailed valuable benefits that help us gain an insight into the changes in the reservoir and the status of the fracturing operation.

1. Identification of any operational issue (e.g. casing integrity, packer integrity)

2. Determination of basic fracture network geometry (i.e. network height, width, half-length)

3. Identification of fracture barriers (e.g. upper, lower, and lateral)

4. Identification of activated faults (common occurrence in the Sichuan basin)

5. Identification of geo-hazards (e.g. water-bearing formations, faults)

6. Enhancement of hydraulic fracturing operation

The paper describes the different fracturing responses between the shale and tight sand, reveals its special characteristic of the natural fracture which activated by fracturing and probably provides the method (Relative magnitude), suitable analysis (Radiated pattern, Linear distribution), and interpretation (Integrated fracture interpretation) to detect the natural fracture through MSM and prevent induced fracture propagation towards fault zones.

\section{The importance of MSM in natural fault detection}

Sichuan is located in the east of the Qinghai-Tibet Plateau and has a strong local stress character and complex fault distribution. There are many distortions in the surface in the Sichuan basin with a northeast trend and there are many faults in the different layers. Although the structural character of surface breaks and the distribution characteristics of the surface faults become the direct basis for subsurface research, it can only be used to reflect the shallow layer characteristics of the energy release and do not comply with the reservoir because of the deep burial of the reservoir in Sichuan. Because of the seismic resolution, some of the faults are neglected, which could have a significant effect on the fracturing operations and exploration. For example, undetected natural faults may connect to the reservoir and the geo-hazards layer and result in the water flooding. Much information of big faults is acquired by 3D seismic or logging, but now many new observations of small faults near to the reservoir could be obtained by the MSM data, even when there is no reflection in 3D seismic or logging data. In recent years, MSM has evolved into a better way to do real-time observation of the activated fault during the fracturing and change the fracturing operation to avoid natural faults and decrease the risk to exploration. Even though it is very important and common to use MSM to detect faults , the question of how to use the MSM data to reliably recognize and confirm a new natural fault has become a complicated issue because of the different characteristics of natural faults and the location error of the MSM.

\section{The characteristics of the natural fault with MSM observation}

The earthquake activation profile has a close relationship to the structure's stress regime and energy. The earthquake always occurs in the weak zone of the energy area and is stimulated along the local maximum stress direction. So also is the behavior of the MSM earthquake during fracturing. The MSM activity is a representation of the geomechanical deformation caused by the hydraulic fracture stimulation. Stimulation designs vary widely with the injection of various fluids and proppant materials of various types (intended to keep the fractures open after the injection stops), and at various injection rates and treating pressures. At the start of the stimulation, increasing the treating pressure results in very low injection rates until the pressure reaches a tensile stress slightly exceeding the minimum principal stress, at which time the injection rate suddenly and rapidly increases at an approximately constant pressure (the so-called breakdown pressure) and activates a micro-fracture and forms a MSM event. In the shale gas reservoir in the Sichuan basin, it is commonly different 


\section{Amsterdam ' 14}

from a tight sand because of the different shape of the fractures. The MSM cloud forms a complex network fracture in shale and a linear-shaped MSM cloud means the probability of a natural fault. Meanwhile, in most of the tight sand reservoirs in Sichuan, the MSM cloud is usually quite different from the other fields because of most of the MSM cloud is located near to the wellbore and very rarely forms a linear-shaped fracture. Thus it is apparently obvious to differentiate a fracturing fracture and a natural fault which has been activated but nobody can confirm it because of different fracturing probably resulting in different response. Nevertheless, there are many other characteristics of the fracturing fracture to differentiate it from the natural fault through MSM data, such as its linear tendency, bigger magnitude, approximate radiation pattern and so on.

\section{Case study}

The treatment well is a horizontal well in the shale gas reservoir. Through the MSM data processing, there are plenty of the MSM events observed with a linear distribution. Through real-time interpretation of the locations of the 4 stages and a natural fault near to the bottom of the treatment well, at the conclusion of $4^{\text {th }}$ stage, the fracturing engineer decided to increase the usage of the silt sand to close off the connection between the fracturing area and the natural fault. The MSM events were stimulated around the perforation of the $5^{\text {th }}$ stage and formed a network fracturing fracture, and less MSM events were stimulated along the natural fault. This enabled the fracturing effect to meet with the fracturing design and to effectively avoid leakage of the fracturing liquid into the natural fault. The following description will give the detailed characteristics of the natural fault activated by fracturing in the Sichuan basin.

Radiated pattern: Similar to a natural fault, the MSM events stimulated in a natural fault have the similar radiated pattern, but it could not be inversed in this case because all the sensors were located in a vertical well, but not scattered around the fracturing area or natural fault in this MSM. Therefore, it is impossible to get the detailed radiated pattern information of the fault to describe the fault activities and detail. However, there has other information to describe the natural fault such as linear distribution, relative magnitude.
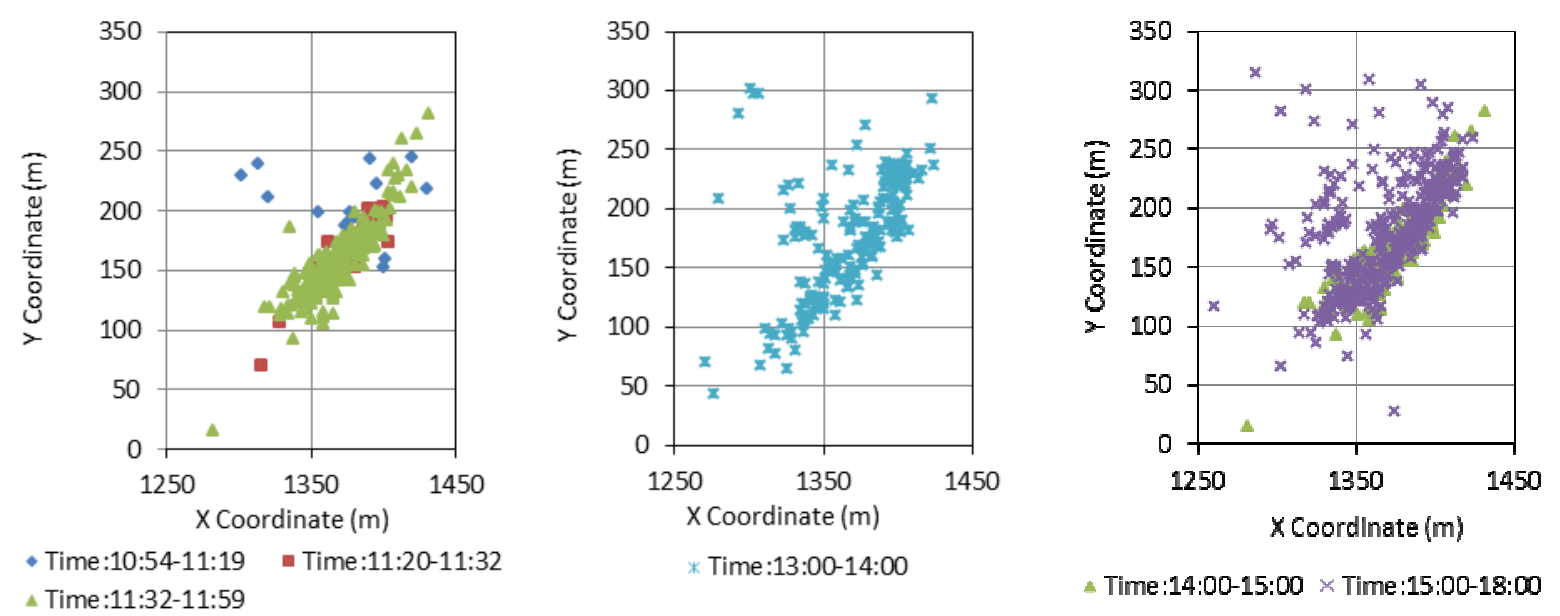

Figure 1 The MSM tendency with different time period in the $2^{\text {nd }}$ stage.

Linear distribution: The MSM events in the $2^{\text {nd }}$ stage are employed to analyse the characteristics which are used to confirm the natural fault. Figure 1 shows the growth of the MSM at different times. At the beginning of the fracturing (10:54 to 11:19), there were several MSM events stimulated which had no linear characteristic. During the (11:20 to 11:32) period of time, there were more MSM events located with a linear distribution and the linear tendency was strengthened during the (11:32 to 11:59) time period. Eventually, most of the MSM events were located with the strong linear distribution. Most of the MSM events in the later time periods, (13:00 to 14:00 and 14:00 to 18:00), were stimulated along the natural fault and formed a stronger linear tendency. 
Relative magnitude: It is commonly considered that larger magnitude MSM events occur along natural faults because they are easier to stimulate. Therefore, it is necessary to invert the MSM magnitude through the wave amplitude and frequency. However, it is difficult to invert a reliable magnitude by the Richter scale because of the differences between MSM and the natural earthquakes, such as the frequency (several times difference), amplitude (thousands to millions times difference), sensor (different response character), geology (different local geology characteristics), and so on. Here, it is possible to invert the relative magnitude based on similar local geology attributes, certain sensor and similar frequency-amplitude responses in the local field. As Figure 2 shows, there are different tendencies in the relative magnitude of the 10 stages. Also before the change in the fracturing operations, the stages from the $1^{\text {st }}$ to the $4^{\text {th }}$ all have a low percentage of magnitudes between -2.5 to -2 . The relative magnitude $(-2$ to -1.5$)$ has a dominant percentage in all the distribution areas and the percentage slowly decreases from the relative magnitude of -1.5 to 1.5 . After the fracturing schedule operations are changed, the stages from the $5^{\text {th }}$ to the $10^{\text {th }}$ have a higher percentage of the relative magnitude area $(-2.5,-1.5)$, and have a sharp decrease in the percentages within the relative magnitude range of -2.5 to 1.5 . Through statistical analysis, before the fracturing schedule changed, the main relative magnitude of the MSM is located in the area -2 to -1.5 , but is in the range of -2.5 to 2 after the fracturing schedule changed. It should be noted that 0.5 times difference in relative magnitude means 16 times difference in energy release. Overall, the relative magnitude in the first 4 stages is generally higher than that in the next 6 stages. Therefore, combining the linear distribution of the MSM events, the relative magnitude and local fracturing fracture character, a natural fault was confirmed.

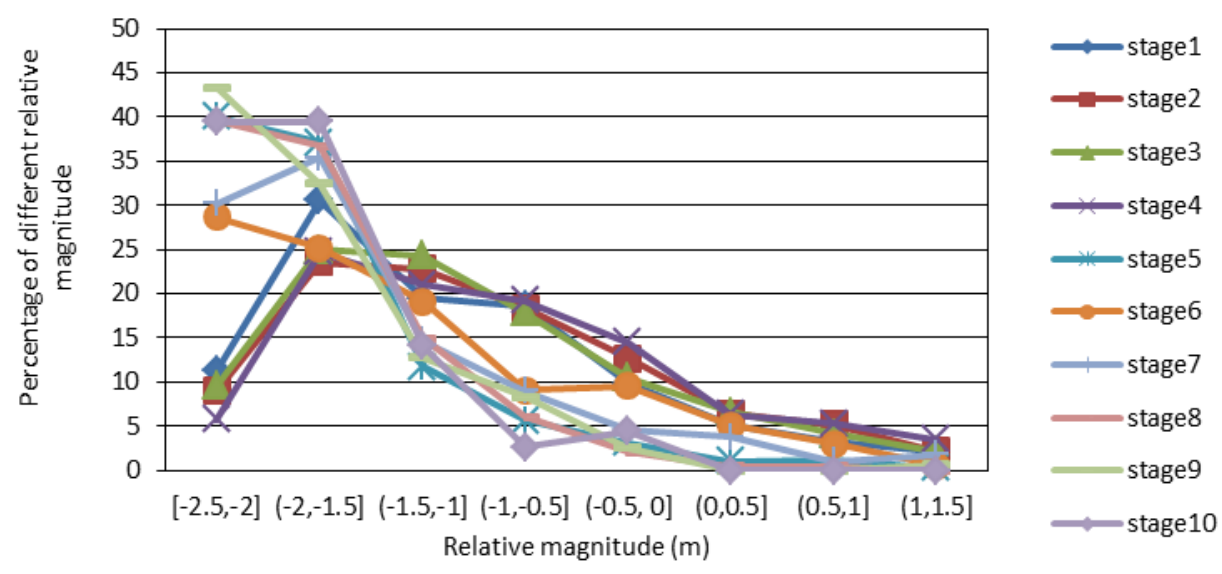

Figure 2 The relative magnitude distribution in the different stages.

Integrated fracture interpretation: Figure 3 shows the fracturing fracture geometry according to the MSM data. Integrating the information from (a), (b) and (c) in every stage, we make the following inferences: (1) The natural fault has an angle of 70 degrees with the horizontal well in the clockwise direction and the depth of the natural fault increases with the distance from the horizontal and grew into the limestone under the reservoir. (2) With the increase in the pumping pressure with the progression of the fracturing, it becomes more difficult to fracture because most of the fracturing liquid leaked to the limestone through the natural fault. (3) In Stage 4, the fracturing engineer suggested to skip the perforation and improve the usage of the silt in the initial phase of the fracturing in the each of the subsequent stages and have close communication between the fracturing engineer and MSM processing engineer to monitoring the change in the reservoir. (4) After the fracturing operation changed in Stage 4, fewer MSM events were stimulated along the natural fault because the silt sand restrained the flow of the fracturing liquid and proppant into the natural fault and many MSM events were stimulated near the perforation and overlapped with the previous the network fracture. (5) The fracturing fracture is not symmetrical but tends to the right side of the horizontal well and the main fracture in each stage is perpendicular to the horizontal well and formed a network fracture. (6) From Stage 5, most of the MSM events were stimulated in the reservoir and have an overlap with the previous stage. 


\section{Amsterdam ' 14}

\section{Conclusions}

It is common to monitor the fault activation in the Sichuan basin because of the complex geology structure and the MSM has already played an important role to discover and to assess the probable natural fault based on the high precision MSM location distribution, relative magnitude and radiation pattern. MSM data interpretation is very complex because it involves multiple disciplines such as geophysics, geology, petrophysics, geomechanics and fracturing engineering, as well as well completion in terms of cementation quality. An in-field real-time comprehensive interpretation needs the multi-disciplinary knowledge. Sometimes the lack of MSM events or MSM events occurred at unexpected locations raising doubts when making decisions on modification to the next fracturing stage based on the current monitoring result. The MSM results give us information about the subsurface and the fracturing induced response but we need to consider various factors to find the hidden information. With the integrated study from various disciplines, the integrated MSM analysis provides reliable understanding for the fracturing operation and well placement plan.

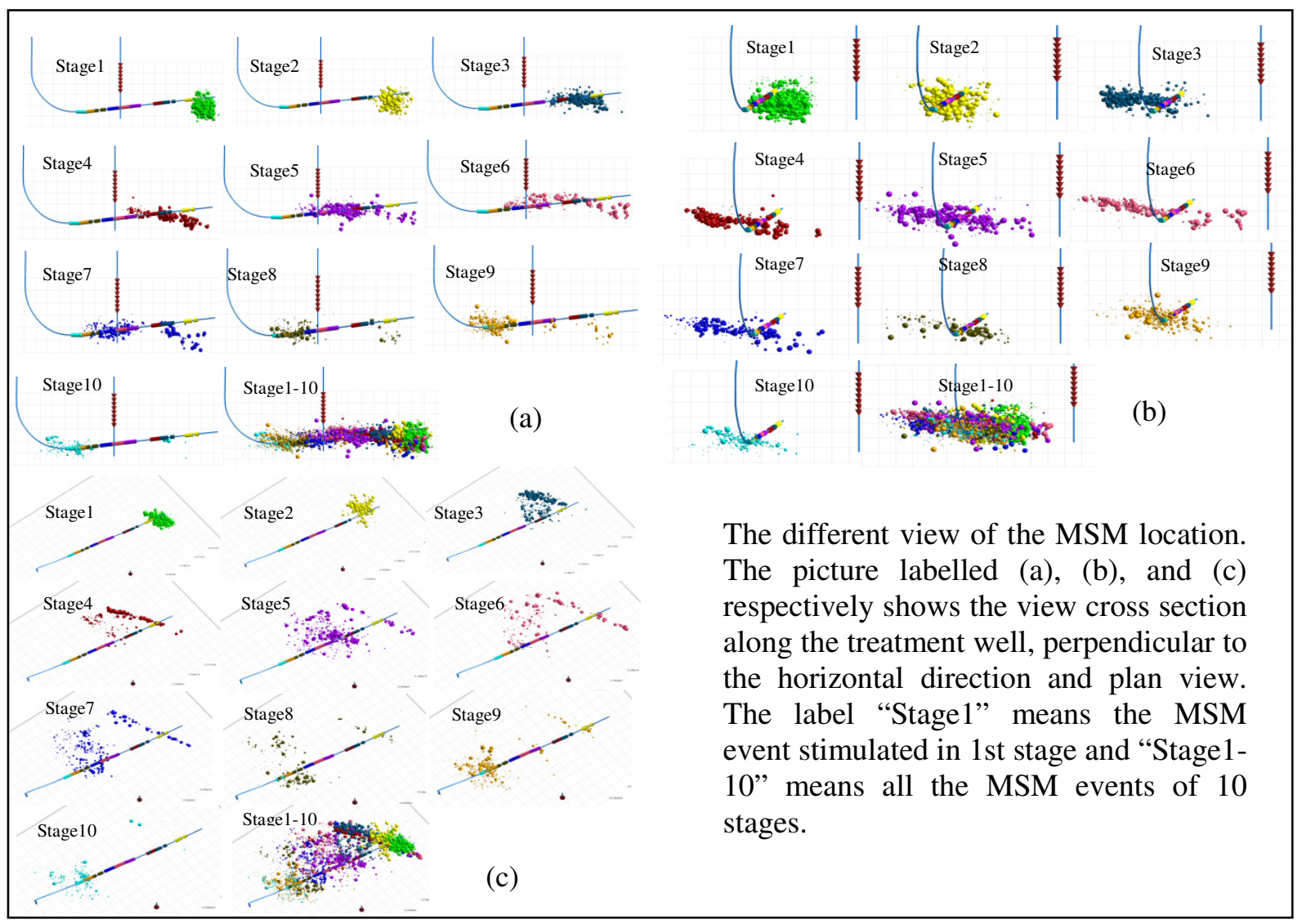

Figure 3 The different view of MSM location of all the stages.

\section{Acknowledgements}

We thank SCGC for the permission to publish this paper.

\section{References}

Yin, C., Liu, H., Wu, F.R., Li, Y.L. et al. [2013] The effect of the calibrated velocity on the microseismic event location precision. SEG Houston Annual Meeting, 21-27 September, 2013. 19771981.

Yin, C., Liu, H., Li, Y.L. et al. [2013] The precision analysis of the microseismic location: Progress in Geophys, 28 (2):0800-0807. 